\title{
BRAND - Search for BSM physics at TeV scale by exploring transverse polarization of electrons emitted in neutron decay
}

\author{
K. Bodek ${ }^{1, \text { a }}$, L. De Keukeleere ${ }^{3}$, M. Kolodziej ${ }^{1}$, A. Kozela ${ }^{2}$, M. Kuzniak ${ }^{5}$, K. Lojek $^{1}$, M. Perkowski ${ }^{1,3}$, H. Przybilski ${ }^{2}$, \\ K. Pysz ${ }^{2}$, D. Rozpedzik ${ }^{1}$, N. Severijns ${ }^{3}$, T. Soldner ${ }^{4}$, A.R. Young ${ }^{6}$, and J. Zejma ${ }^{1}$ \\ ${ }^{1}$ M. Smoluchowski Institute of Physics, Jagiellonian University, Cracow, Poland \\ ${ }^{2}$ H. Niewodniczanski Institute of Physics, Polish Academy of Sciences, Cracow, Poland \\ ${ }^{3}$ Institute of Nuclear and Radiation Physics, KU Leuven, Belgium \\ ${ }^{4}$ Institut Laue-Langevin, Grenoble, France \\ ${ }^{5}$ Carleton University, Ottawa, Canada \\ ${ }^{6}$ Department of Physics and Astronomy, North Carolina State University, Raleigh, USA
}

\begin{abstract}
Neutron and nuclear beta decay correlation coefficients are linearly sensitive to the exotic scalar and tensor interactions that are not included in the Standard Model (SM). The proposed experiment will measure simultaneously 11 neutron correlation coefficients $(a, A, B, D, H, L, N, R, S, U, V)$ where 7 of them $(H, L, N, R, S, U, V)$ depend on the transverse electron polarization - a quantity that vanishes for the SM weak interaction. The neutron decay correlation coefficients $H, L, S, U, V$ were never attempted experimentally before. The expected ultimate sensitivity of the proposed experiment that currently takes off on the cold neutron beamline PF1B at the Institut Laue-Langevin, Grenoble, France, is comparable to that of the planned electron spectrum shape measurements in neutron and nuclear $\beta$ decays but offers completely different systematics and additional sensitivity to imaginary parts of the scalar and tensor couplings.
\end{abstract}

\section{Introduction}

Nuclear and neutron beta decay have played a crucial role in the development of the weak interaction theory. Among the empirical foundations of the electroweak standard model (SM) the assumptions of maximal parity violation, vector and axial-vector character and massless neutrinos are directly linked to the experiments performed with the nuclear and neutron beta decay. The beta decay theory has been firmly established about four decades ago and became a part of the SM. It describes the semi-leptonic and strangeness conserving processes in the 1-st particle generation mediated by the charged $W$-boson exchange. In course of time, only the neutrino mass appeared to be finite the beta decay experiments with increasing precision still confirm the first two assumptions. Nevertheless, despite the great success of the SM, many open questions remain such as the origin of parity violation, the hierarchy of fermion masses, the number of particle generations, the mechanism of $\mathrm{CP}$ violation, the worrying large number of parameters of the theory, etc. Presently, the main aim of low-energy experiments such as beta decay is to find deviations from the SM assumptions as possible indications of new physics.

Intensive searches for new physics beyond the Standard Model (BSM) concentrate on two main frontiers: (i) high energy experiments performed at collider accelerators and (ii) low energy precision experiments.

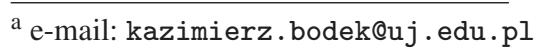

While the first group looks for exotic particles produced on-shell in high energy collisions, the second one seeks for tiny deviations in low energy observables which can be attributed to non-existent in the SM exotic interactions. Nuclear and neutron beta decays are prominent examples of the second group. The persistent problem connected with this duality is to establish a common language in order to compare the results and allow for combined analyzes. Presently, the best solution is offered by the effective field theory (EFT) which can be safely applied to both experimental domains as long as the scale for exotic phenomena is sufficiently higher than the energy achievable in accelerators. In a number of seminal papers [1-6] the EFT projection was applied to the charged current (CC) weak interaction including yet not observed but allowed by Lorentz invariance genuine scalar and tensor contributions. The EFT parametrization of measured observables in nuclear, neutron and pion decay and of the cross section for the missing transverse energy channel (MET) $p p \rightarrow e \bar{v}+M E T+\ldots$ at high transverse mass led to the combined limits for exotic scalar and tensor interactions showing that, presently, the constraints obtained from both approaches are on a comparable accuracy level. Moreover, the EFT analysis describes prospects and defines challenges for future experiments in the low- and high-energy searches for BSM physics.

The above described parametrization and analysis was applied to the observables measured in the past and addressed in ongoing and prepared experiments. In this paper, we recall and elaborate on the idea of 
an entirely new experiment devoted to a simultaneous measurement of 11 correlation coefficients in the neutron decay where 5 of them were never attempted before [7]. Seven of these correlation coefficients are related to the transverse polarization component of the electrons emitted in the decay. The proposed experiment offers a potential sensitivity comparable to that of the "classical" correlation experiments and completely different systematics. Control of systematic effects is the key issue for precision experiments.

\section{Neutron $\beta$-decay}

An exhaustive list of merits of the neutron physics and the role the neutron plays in our understanding of universe can be found in recent review papers and in rich references therein [8-10]. The neutron beta decay belongs to the electroweak sector of the neutron physics. It is an allowed and mixed transition. Contrary to the nuclear mixed beta transitions, the Fermi and Gamow-Teller contributions to the neutron decay rate and energy-angle distribution are known accurately since the corresponding matrix elements are not affected by nuclear structure uncertainties. In the SM, the neutron beta decay is described by only two parameters: $V_{u d}$ element of the Cabibo-KobayashiMaskawa mixing matrix and the ratio of the axial vector to vector charges, $\lambda=g_{A} / g_{V}$. The large number (already in the first order) of the experimental observables depending on the momenta and spins of the involved particles makes the situation highly redundant and attractive for precision experiments which, on the one hand, improve the SM and, on the other hand, search for the effects which cannot be explained by the SM. At low energy, the domain natural for beta decay, the tests of the SM generally refer to tests of the underlying fundamental symmetries rather than to tests of consistency of theory or predictions of new phenomena. In this sense, the found deviations from the SM symmetry assumptions can be treated as possible indications of new physics. For instance, the SM predictions of time-reversal violation for weak decays of systems built up of $u$ and $d$ quarks are by 7 to 10 orders of magnitude lower than the experimental accuracies attainable at present. It is a general presumption that time-reversal phenomena are caused by a tiny admixture of exotic interaction terms. Unfortunately, the exploration of this intriguing window for new physics is mitigated by the accuracy of theoretically calculated final state effects (FSI) which presently achieve the level slightly better than $10^{-4}[11,12]$. Nevertheless, weak decays provide a favorable testing ground in a search for such feeble forces.

\subsection{Transverse electron polarization in neutron decay}

Generally, in the Standard Model, the electrons emitted in beta decay are longitudinally polarized which reflects the parity violating V-A structure of the weak interaction. The departure of the polarization vector from a strict collinearity with electron momentum can be caused by electromagnetic effects, recoil order corrections (induced couplings) or genuine scalar and tensor interactions. Thus, provided that the electromagnetic and recoil order corrections are under control, the transverse electron polarization is an ideal observable for searches of physics beyond the Standard Model. The additional feature of the neutron decay is that it is a mixed transition and is sensitive to both genuine scalar and tensor couplings at once.

The transverse electron polarization is reflected in the distribution of the decay products via a number of the correlation coefficients relating it to other vectors characterizing the system, the most important being the electron and antineutrino momenta $\mathbf{p}_{e}, \mathbf{p}_{\bar{v}}$ and the neutron spin $\mathbf{J}$. The corresponding formula limited to the lowest order terms can be found in the classical papers [13-15]. Dropping out all the terms not depending explicitly on the transverse components of the electron polarization and retaining five exceptions: $a, b, A, B$ and $D$ ("classical" correlations) one arrives at:

$$
\begin{aligned}
& \omega\left(E_{e}, \Omega_{e}, \Omega_{\bar{v}}\right) \propto 1+a \frac{\mathbf{p}_{e} \cdot \mathbf{p}_{\bar{v}}}{E_{e} E_{\bar{v}}} \\
& +b \frac{m_{e}}{E_{e}}+\frac{\langle\mathbf{J}\rangle}{J} \cdot\left[A \frac{\mathbf{p}_{e}}{E_{e}}+B \frac{\mathbf{p}_{\bar{v}}}{E_{\bar{v}}}+D \frac{\mathbf{p}_{e} \times \mathbf{p}_{\bar{v}}}{E_{e} E_{\bar{v}}}\right] \\
& +\sigma_{\perp} \cdot\left[H \frac{\mathbf{p}_{\bar{v}}}{E_{\bar{v}}}+L \frac{\mathbf{p}_{e} \times \mathbf{p}_{\bar{v}}}{E_{e} E_{\bar{v}}}+N \frac{\langle\mathbf{J}\rangle}{J}+R \frac{\langle\mathbf{J}\rangle \times \mathbf{p}_{e}}{J E_{e}}\right. \\
& \left.\quad+S \frac{\langle\mathbf{J}\rangle}{J} \frac{\mathbf{p}_{e} \cdot \mathbf{p}_{\bar{v}}}{E_{e} E_{\bar{v}}}+U \mathbf{p}_{\bar{v}} \frac{\langle\mathbf{J}\rangle \cdot \mathbf{p}_{e}}{J E_{e} E_{\bar{v}}}+V \frac{\mathbf{p}_{\bar{v}} \times\langle\mathbf{J}\rangle}{J E_{\bar{v}}}\right],
\end{aligned}
$$

where $\sigma_{\perp}$ represents a unit vector perpendicular to the electron momentum $\mathbf{p}_{e}$ and $J=|\mathbf{J}|$. In the infinite neutron mass approximation (no recoil), making usual assumptions for the Standard Model: $C_{V}=C_{V}^{\prime}=1$ and $\lambda \equiv C_{A}=$ $C_{A}^{\prime}=-1.272$ [16], and neglecting the contributions quadratic (and higher order) in $C_{S}, C_{S}^{\prime}, C_{T}, C_{T}^{\prime}$ one can express all the correlation coefficients from Eq. (1) (called here $X$ ) as combinations of the real and imaginary parts of the scalar, $\mathfrak{S}$, and tensor, $\mathfrak{T}$, exotic couplings:

$$
\begin{aligned}
X= & X_{\mathrm{SM}}+X_{\mathrm{EM}}+c_{\mathrm{Re} S}^{X} \operatorname{Re}(\mathfrak{S})+c_{\mathrm{Re} T}^{X} \operatorname{Re}(\mathfrak{T}) \\
& +c_{\operatorname{Im} S}^{X} \operatorname{Im}(\mathfrak{S})+c_{\operatorname{Im} T}^{X} \operatorname{Im}(\mathfrak{T})
\end{aligned}
$$

with

$$
\mathfrak{S} \equiv \frac{C_{S}+C_{S}^{\prime}}{C_{V}}, \quad \mathfrak{T} \equiv \frac{C_{T}+C_{T}^{\prime}}{C_{A}} .
$$

The coefficients $c$ in this expression are functions of $\lambda$ and kinematical quantities. Table 1 summarizes their values calculated with the kinematical factors averaged over the electron spectrum in the kinetic energy range 200-783 keV. $X_{S M}$ is the SM value of $X$ and the electromagnetic corrections called $X_{E M}$ were calculated in the static Coulomb field approximation with point-like proton and including only the contributions linear in the fine structure constant $\alpha[14,15]$.

The coefficients relating the transverse electron polarization to $\mathbf{p}_{e}, \mathbf{p}_{\bar{v}}$ and $\mathbf{J}$ have several interesting features. They vanish for the SM weak interaction, reveal variable size of the EM contributions and are measurable in the present experiments. And, last but not least, the dependence on real and imaginary parts of the scalar and tensor couplings alternates exclusively from one correlation coefficient to another with varying sensitivity coefficients. This feature allows one to deduce a complete set of constraints for $\mathfrak{S}$ and $\mathfrak{T}$ from the neutron decay alone. However, as pointed out in Refs. [2,4], there appears a serious technical problem potentially leading to 
Table 1. Sensitivity factors for scalar and tensor couplings (leading order, no recoil, point charge). Calculations were performed with $\lambda=-1.272$ and kinematical factor averaged over electron kinetic energy $E_{\mathrm{k}}=(200,783) \mathrm{keV}$. This is an updated version of Table 2 from Ref. [7].

\begin{tabular}{ccccccc}
\hline \hline$X$ & $X_{S M}$ & $X_{E M}$ & $c_{\mathrm{Re} S}^{X}$ & $c_{\mathrm{Re} T}^{X}$ & $c_{\operatorname{Im} S}^{X}$ & $c_{\operatorname{Im} T}^{X}$ \\
\hline$a$ & -0.1048 & 0 & $-0.1714^{*}$ & $-0.1714^{*}$ & -0.0007 & +0.0012 \\
$b$ & 0 & 0 & +0.1714 & +0.8283 & 0 & 0 \\
$A$ & -0.1172 & 0 & 0 & 0 & -0.0009 & +0.0014 \\
$B$ & +0.9876 & 0 & -0.1264 & +0.1945 & 0 & 0 \\
$D$ & 0 & 0 & +0.0009 & -0.0009 & 0 & 0 \\
$H$ & 0 & +0.0609 & -0.1714 & +0.2762 & 0 & 0 \\
$L$ & 0 & -0.0004 & 0 & 0 & +0.1714 & -0.2762 \\
$N$ & 0 & +0.0668 & -0.2176 & +0.3348 & 0 & 0 \\
$R$ & 0 & +0.0005 & 0 & 0 & -0.2176 & +0.3348 \\
$S$ & 0 & -0.0018 & +0.2176 & -0.2176 & 0 & 0 \\
$U$ & 0 & 0 & -0.2176 & +0.2176 & 0 & 0 \\
$V$ & 0 & 0 & 0 & 0 & -0.2176 & +0.2176 \\
\hline \hline
\end{tabular}

${ }^{*}\left(\left|C_{S}\right|^{2}+\left|C_{S}^{\prime}\right|^{2}\right) / 2$ instead of ReS and $\left(\left|C_{T}\right|^{2}+\left|C_{T}^{\prime}\right|^{2}\right) / 2$ instead of ReT, respectively.

suppression or even to complete loss of linear sensitivity to the exotic couplings since, instead of $X$, effectively, measurements deliver $\widetilde{X}=X /\left(1+b \frac{m}{E_{e}}\right)$. Expanding $\widetilde{X}$ in small exotic coupling constants and retaining only linear terms gives:

$$
\begin{aligned}
\widetilde{X}= & \frac{X}{1+b \frac{m}{E_{e}}} \approx X_{\mathrm{SM}}+X_{\mathrm{EM}} \\
& +\left[c_{\mathrm{Re} S}^{X}-c_{\mathrm{Re} S}^{b}\left(X_{\mathrm{SM}}+X_{\mathrm{FSI}}\right)\right] \operatorname{Re}(\mathfrak{S}) \\
& +\left[c_{\operatorname{Re} T}^{X}-c_{\operatorname{Re} T}^{b}\left(X_{\mathrm{SM}}+X_{\mathrm{FSI}}\right)\right] \operatorname{Re}(\mathfrak{T}) \\
& +c_{\mathrm{Im} S}^{X} \operatorname{Im}(\mathfrak{S})+c_{\operatorname{Im} T}^{X} \operatorname{Im}(\mathfrak{T})
\end{aligned}
$$

Fortunately, the transverse electron polatization related coefficients are safe with that respect: the cancelation term is proportional to $\left(X_{\mathrm{SM}}+X_{\mathrm{EM}}\right)$ which even in the extreme cases of large electromagnetic contribution $(H, N)$ does not exceed $0.07[11,12,14,15]$ so that the potential loss of the linear sensitivity to the real part of the scalar and tensor couplings is significantly suppressed. This feature makes the transverse electron polarization related coefficients attractive for future searches of BSM physics.

\subsection{EFT parametrization}

In order to bridge the classical $\beta$-decay formalism with high-energy physics and permit sensitivity comparison of low-energy charged-current observables with measurements carried out at high-energy colliders, the modelindependent EFT framework is employed. The effective nucleon-level couplings $C_{i}, C_{i}^{\prime}(i \in[V, A, S, T])$ can be generally expressed as combinations of the quark-level parameters $\epsilon_{i}, \tilde{\epsilon}_{i}(i \in[L, R, S, T])$ [4]. The imaginary parts of the scalar and tensor couplings parametrize CPviolating contributions. The high energy BSM physics process that can be compared with $\beta$-decay experiments is the cross section for electrons and missing transverse energy $(\mathrm{MET})$ in $p p \rightarrow e \bar{v}+M E T+\ldots$ channel since it has the same underlying partonic process as in $\beta$-decay $(\bar{u} d \rightarrow e \bar{v})$. With the anticipated accuracy of
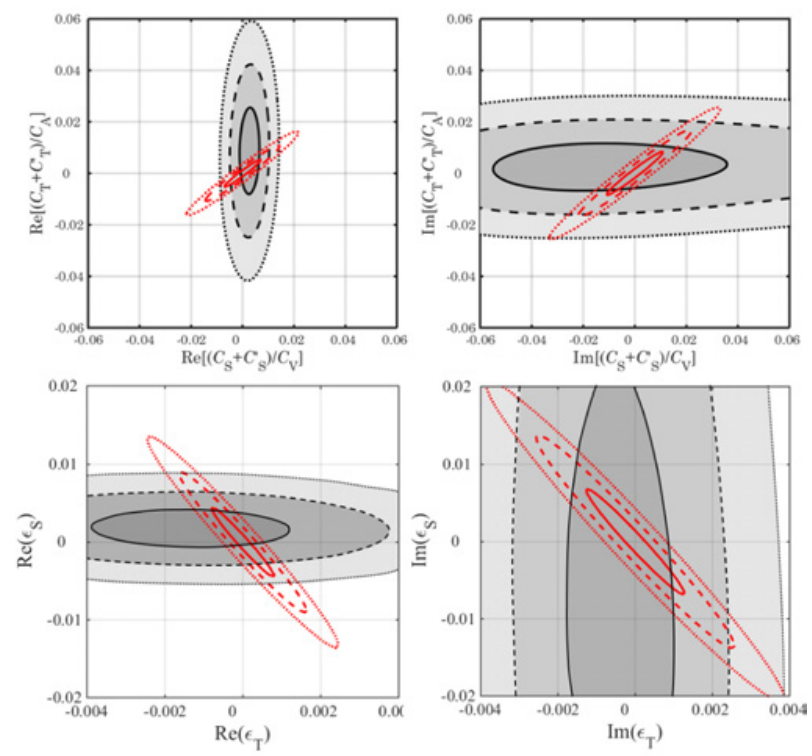

Figure 1. Experimental bounds on the scalar vs. tensor couplings $\mathfrak{S}, \mathfrak{T}$ from Eq. (3) (upper panels) and translated to EFT parameters $\epsilon_{S}, \epsilon_{T}$ (lower panels). The gray areas represent the information deduced from presently available experiments, while the red lines represent the limits resulting from the correlation coefficients $H, L, N, R, S, U$ and $V$ measured with the anticipated accuracy of $5 \times 10^{-4}$. Solid, dashed and dotted lines correspond to $1-, 2-$ and $3-\sigma$ confidence levels, respectively, in analogy to decreasing intensity of the grey areas.

about $5 \times 10^{-4}$ for the transverse electron polarization related correlation coefficients one will obtain significantly tighter bounds on the real and imaginary parts of $\mathfrak{S}$ and $\mathfrak{T}$ and, consequently, on $\epsilon_{S}$ and $\epsilon_{T}$ as shown in Fig. 1 adopted from the graphs included in Fig. 5 of Ref. [7] and Fig. 2 of Ref. [17]. It should be noted that such limits would be competitive to those extracted from the analysis $20 \mathrm{fb}^{-1} \mathrm{CMS}$ collaboration data collected at $8 \mathrm{TeV}[18,19]$ and even to the planned measurements at $14 \mathrm{TeV}$. 
The anticipated experimental bounds on the scalar and tensor couplings delivered by the leptoquark exchange and MSSM models can be found in Ref. [7].

\section{Experiment}

The experiment reported in Refs. [7,20-22] has clearly proven that the transverse electron polarization of electrons emitted in the decay of cold neutrons can be accurately measured with Mott scattering. The applied technique of electron tracking and reconstruction of the scattering vertices is a powerful tool for reduction of background typical in experiments with intense neutron beams. Electron tracking suppresses many difficulties connected to the large dimensions of the decay source. The rich experience and quantitative information gained in that pioneering experiment allow for a realistic planning of the next steps. The main factors limiting the achieved accuracy were: (i) small angular coverage of the detecting system, (ii) relatively weak, divergent and poorly polarized cold neutron beam as compared to present possibilities and (iii) transport of neutrons in helium gas which allowed for simple and very thin $(\geq 2.5 \mu \mathrm{m}$ Mylar) detector windows but, simultaneously, contributed significantly to the background increase since about $3 \%$ of beam neutrons scattered from helium were captured in detectors and surroundings. The idea described in the following incorporates not only the conclusions from the first experiment but implements new features which make the proposed experimental setup more versatile and powerful.

The proposed experiment will measure 11 correlation coefficients ( $a, A, B, D, H, L, N, R, S, U, V)$ as compared to three $(A, N, R)$ accessible in the former setup. Obviously, the simultaneous measurements of the coefficients $a, A, B$ and $D$ will help to keep systematics under control. In further perspective it will be very intriguing to compare these results with those by the specialized experiments [23-30] since our experimental technique is completely different from that applied in the above mentioned experiments. Also different are the associated systematic effects.

The strategy of the proposed experiment is to reconstruct event-by-event the decay kinematics and identify the decay origin which must be localized in the beam fiducial volume. Such a condition will reduce the effects due to large size of the decay source and significantly suppress background. In particular, fixing the three-body decay kinematics by the measured electron energy and relative $e-p$ angle one realizes that the proton energy and thus the proton time-of-flight must choose between two discrete (and known) values. The measurement of momenta of electrons and protons in coincidence allows for reconstruction of the antineutrino momentum and provides access to the terms dependent on this quantity.

\subsection{BRAND setup}

The transverse polarization of electrons will be analyzed in the backward angle Mott scattering on high $Z$ nuclei (e.g. $\mathrm{Pb}$ or $\mathrm{U}$ ). The attractiveness of this method arises not only from the exceptionally large analyzing power (approaching 0.5 at backward angles). Mott scattering is governed by the time reversal and parity conserving electromagnetic interaction and thus it is insensitive (on the level of $10^{-7}$ ) to the fake effects (e.g. geometry misalignment) from the longitudinal electron polarization dominating in $\beta$-decay. This feature is unique among the measurements of the transverse polarization of leptons. It has been demonstrated in the pioneering experiment that this technique works nicely [7,20-22] and its potential has not yet been exhausted. The achieved respectably small systematic uncertainty of $5 \times 10^{-3}$ for the $R$ coefficient and $4 \times 10^{-3}$ for $N$ can be improved by at least an order of magnitude which follows from conservative evaluation of the proposed methods. Due to the increase of the beam intensity and angular acceptance of detectors the expected gain in the rate of triggered events should be greater than 300 . Besides of that, one expects significantly larger neutron polarization $(>0.99)$ and the Mott target analyzing power (in case of depleted uranium target).

The key features of the proposed setup are: (i) Efficient cylindrical detector geometry. (ii) Electron tracking in a multi-wire drift chamber (MWDC) with signal readout at both wire ends. (iii) Detection of both direct and Mottscattered electrons in a plastic scintillator hodoscopes. (iv) Conversion of protons (accelerated to $20-30 \mathrm{keV}$ ) into bunches of electrons ejected from a thin $\mathrm{LiF}$ layer [32]. Acceleration and subsequent detection of ejected electrons in a thin $(\sim 25 \mu \mathrm{m})$ plastic scintillator with position sensitivity. (v) Reduction of geometry misalignment related systematic effects by the use of advanced polarimetry techniques such as periodic neutron spin flip during data taking and evaluation of asymmetry and super-ratio in data analysis. Figure 2 illustrates the sensitivity maps (as functions of kinematical variables) for the transverse electron polarization related correlation coefficients. (vi) Background contributions will be subtracted using data collected in several regimes (beam-on, beam-off, Mott target-on, Mott target-off). (vii) In order to deduce necessary corrections and asses associated systematic errors, mapping of the spin guiding field and detector efficiency will be carried out in a series of calibration experiments.

The cylindrical geometry was already considered in the context of the $R$ correlation coefficient measurement [33] but not attempted as too challenging for a pioneering experiment. The detection of recoil protons in conjunction with complete reconstruction of the electron tracks is a feature attempted only in the TRINE experiment [31]. A cross section of the proposed cylindrical Mott polarimeter is sketched in Fig. 3. The cold neutron beam will be polarized longitudinally ( $a, H$ and $L$ coefficients prefer unpolarized beam). In further perspective the transverse neutron polarization is considered, too. The vacuum of the decay volume will be separated from the electron detection section with a few $\mu \mathrm{m}$ thick Mylar foil stretched over a rigid cylindrical structure consisting of a perforated metal (Al) tube with a thin Kevlar thread wound on it similar as described in Ref. [34]. The window area will cover about $50 \%$ of the solid angle available for electrons from neutron decay.

The electron tracking detector will consist of a 6-7 layer multi-wire drift chamber (MWDC) with hexagonal geometry of cells operated in He/isobutane gas mixture. Decay electrons are tracked in MWDC and reach the Mott target made of 1-2 $\mu \mathrm{m}$ thick lead (or depleted uranium) layer deposited on a thin Mylar substrate. 

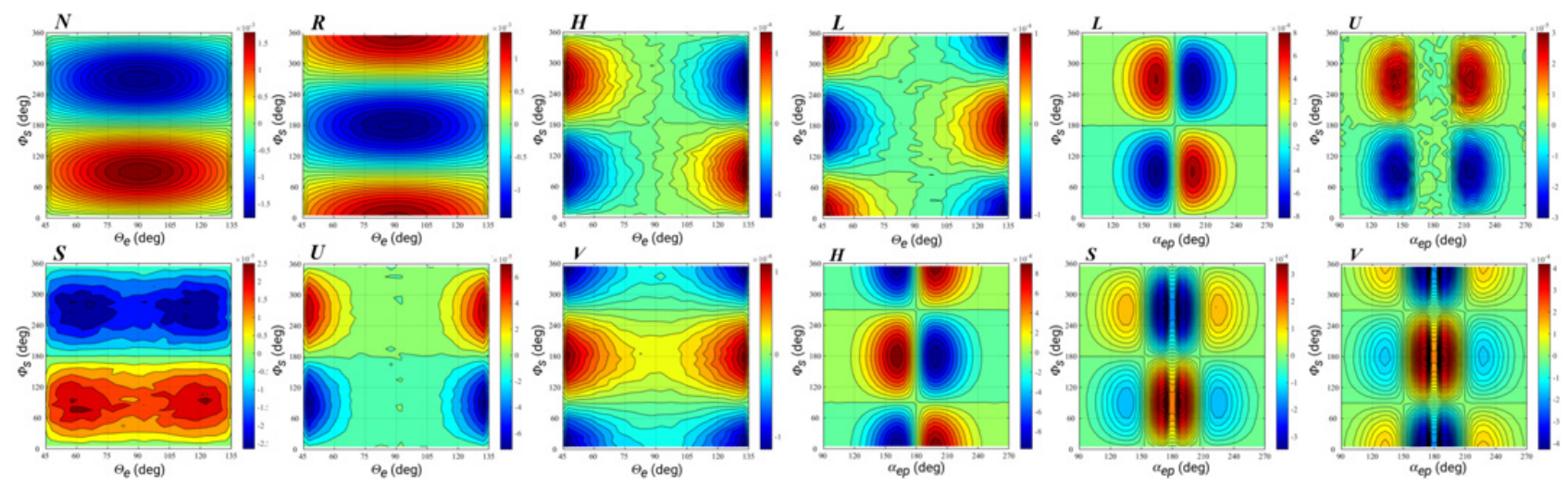

Figure 2. Sensitivity maps for the $N, R, H, L, S, U$ and $V$ coefficient as a function of the polar electron angle $\theta_{e}$ or the relative electron-proton angle $\alpha_{e p}$ and the azimuthal spin projection angle $\phi_{s}$ (arbitrary units). The kinematical acceptance is defined by $E_{e}^{\mathrm{kin}} \in(200,782) \mathrm{keV}, E_{p}^{\mathrm{kin}} \in(50,800) \mathrm{eV}, \theta_{e} \in\left(45^{\circ}, 135^{\circ}\right), \theta_{p} \in\left(30^{\circ}, 150^{\circ}\right)$.

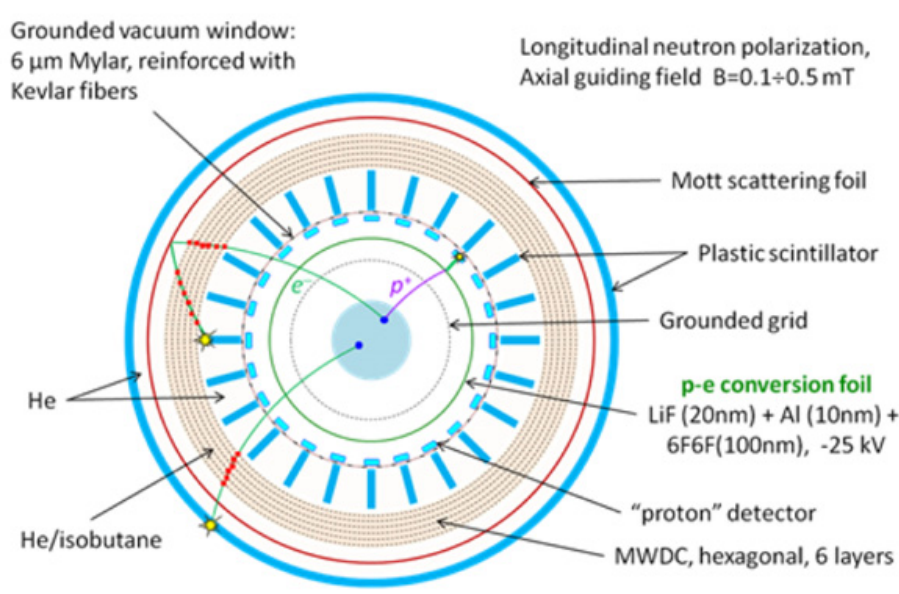

a)

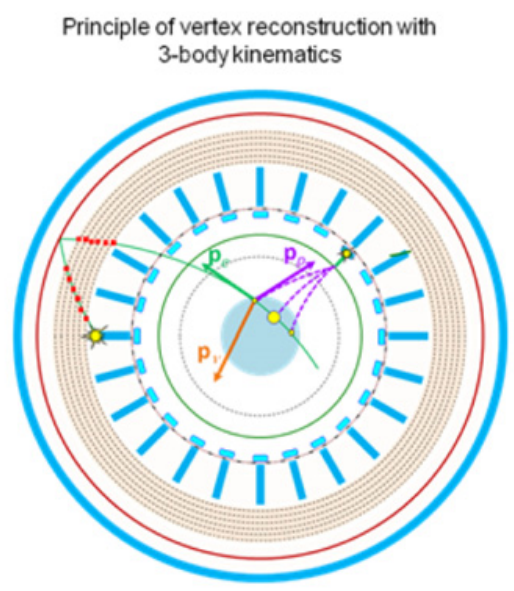

b)
Figure 3. Sketch of the proposed experimental setup in the cylindrical geometry. a) Cross section perpendicular to the beam (and detector) axis. b) Illustration of the conditional reconstruction of the 3-body kinematics using the likelihood method. Size of the yellow dots represent the likelihood assigned to particular solutions which is proportional to the neutron decay density in the fiducial volume.
The decay electrons can be either backscattered (about 1 in 1000 cases) or pass the foil and be detected in the outer plastic scintillator hodoscope located close to it. The Mott-scattered electrons are registered in either of the inner or the outer hodoscopes as shown in Fig. 3. In this way, the acceptance in the dictated by the Figure-of-Merit optimal angular range $\left(100^{\circ}-140^{\circ}\right)$ is maximal in such a configuration. For direct electrons, the low energy threshold will be about $100 \mathrm{keV}$ taking into account all materials traversed prior to detection. For the Mott scattered electrons, the threshold will rise to about $150 \mathrm{keV}$. This value is satisfactory as the Figureof-Merit for the spin analysis in the Mott process drops down rapidly below the electron kinetic energy of $200 \mathrm{keV}$ (cf. Fig. 4a). For recoil protons, the feasible low energy thresholds of $50 \mathrm{eV}$ is defined by the assumed maximum time-of-flight window of about $1000 \mathrm{~ns}$ (cf. Fig. 4b).

The electron hodoscopes will be made of plastic scintillator bars readout by photomultiplier tubes attached to both bar ends. They will operate at ambient pressure outside of the vacuum chamber.

After leaving the free drift zone, protons are accelerated by electric field towards $p-e$ conversion foil a)

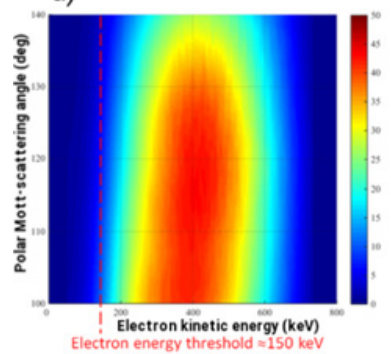

b)

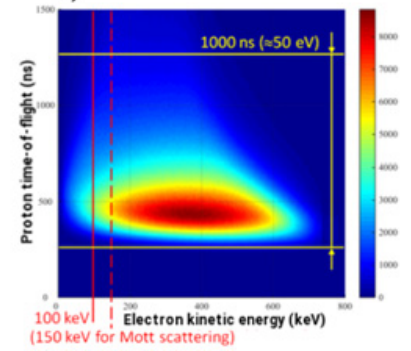

Figure 4. Figure-of-Merit (arbitrary units) for the spin analysis in backward angle Mott scattering on a $2 \mu \mathrm{m}$ thick $\mathrm{Pb}$ target. b) Population of the neutron decay phase space projected onto the proton time-of-flight vs. electron kinetic energy $E_{e}^{\mathrm{kin}}$ plane.

consisting of a $20 \mathrm{~nm}$ thick LiF layer deposited onto a thin $(100 \mathrm{~nm})$ polyamide film [32]. The located in vacuum "proton" detectors will register a bunch of 10-20 electrons ejected from that foil and accelerated to $25-30 \mathrm{keV}$ in the electric field. "Proton" detectors will be made of thin $(25 \mu \mathrm{m})$ plastic scintillator film deposited onto 
light guides. The scintillation light will be registered by SiPM sensors attached along light guide. The hit position information will be deduced from the distribution of light signal registered by SiPM sensors close to the hit position. The "proton" detector will be able to discriminate electrons from beta decay with energy higher than $30 \mathrm{keV}$ since they deposit in it less than $30 \mathrm{keV}$ while the accumulated signal from the electron bunch following the $p-e$ conversion corresponds to $250-500 \mathrm{keV}$.

\subsection{Data acquisition and measurement scenarios}

The key features of the data acquisition will be:

1. Only one trigger condition: registration of an electron in either of the plastic scintillator hodoscopes in coincidence with user selectable hit multiplicity of MWDC. This signal will also give a time reference for all other signals delivered by MWDC and "proton" detectors.

2. Acquisition of all signals generated on wires and scintillators within a time window of about $1 \mu \mathrm{s}$. The drift time will be measured by multi-hit TDC. Pulse height will be measured in parallel with ADC. Two alternative options are also considered: (i) obtaining pulse height information from TDC data using the improved time-over-threshold method and (ii) obtaining both the drift time and pulse height information from signals sampled at frequency of about $500 \mathrm{MHz}$.

3. Signals delivered by SiPM sensors serving "proton" detectors will be acquired by dedicated electronic channels.

Acquired information will allow to deduce: (i) electron energy from pulse height in the scintillator, (ii) proton hit position from the pulse height distribution of light signals registered by SiPM sensor close to hit position, (iii) electron tracks from drift times and pulse height asymmetry on both wire ends in MWDC, (iv) proton energy from measured time-of-flight, hit position and 3body kinematics.

The proposed solutions imply registration of both Mott-scattered and direct (without Mott scattering) electrons and with and without accompanying protons. Fixed 3-body decay kinematics and thus fixed relation between electron energy and proton time-of-flight will allow for a short coincidence time window which reduces significantly accidental coincidences and allows for large single rates of detectors. On the other hand, the fine granularity (5-10 $\mathrm{mm}$ pitch for MWDC) guaranties that even an outstanding neutron decay rate of $10^{5} \mathrm{~s}^{-1}$ in the fiducial volume will cause the pulse rate of less than $1 \mathrm{kHz}$ on a $1 \mathrm{~m}$ long wire. Plastic scintillators applied for electron energy registration are capable to accept the single rate up to $1 \mathrm{MHz}$ with tolerable pileup fraction while "proton" plastic scintillators are expected to experience a counting rate well below $100 \mathrm{kHz}$.

Although, due to the finite size of the beam cross section, the exclusive kinematics can be reconstructed only approximately, the gain of information over the classical "integral" approach is enormous. Conditional reconstruction of the decay kinematics and thus the proton and antineutrino momenta with precisely known weights (decay probability distribution along the reconstructed electron path segment coinciding with the beam is known) is sufficient since for the extraction of the correlation coefficients one must anyhow integrate over momenta.

The experiment will be run in two modes: (1) unpolarized neutrons - measurement of $a, H, L$ and (2) polarized neutrons - measurement of $a, A, B, D$, $H, L, N, R, S, U$ and $V$ coefficients. In the extraction of the correlation coefficients from measured data, the symmetry properties of particular terms with respect to the transformations $\mathbf{J} \rightarrow-\mathbf{J}, \mathbf{p}_{e} \rightarrow-\mathbf{p}_{e}$ and $\mathbf{p}_{\bar{v}} \rightarrow-\mathbf{p}_{\bar{v}}$ will be utilized. It has been estimated that with a detecting system accepting $10^{5}$ decays per second in the $1 \mathrm{~m}$ long fiducial volume and one year long data taking period one can acquire about $5 \times 10^{11}$ direct electrons and $3 \times 10^{8}$ Mott scattered electrons in coincidence with protons. These numbers are sufficient for the anticipated ultimate sensitivity of about $5 \times 10^{-4}$ for the transverse electron polarization related correlation coefficients. Moreover, they assure statistically unbiased measurements of $a, A$, $B$ and $D$ on the level of few times $10^{-5}$.

\section{Systematic effects}

The central issue of BRAND is the transverse electron polarization produced in the neutron decay origin which is the quantity carrying the information about possible scalar and/or tensor admixtures in weak interactions. However, the electron polarization at the place of measurement is slightly different from the original one due to electromagnetic fields influencing the electron momentum and spin on the way from the decay origin to the Mott scattering vertex.

The dominating effects which should be kept under control and corrected for, if necessary, are discussed below.

\subsection{Final state interaction (FSI)}

This correction and its uncertainty is of theoretical origin. It arises from the interaction of the emitted electron with electromagnetic field of the recoil proton. In Table 1, we listed the FSI effects estimated in the lowest order in Refs. [14,15]. A better estimate exists only for the $N$ and $R$ coefficient $[11,12]$. It is expected that the BRAND experiment will stimulate theoreticians to calculate systematically a complete set of FSI corrections for all relevant correlation coefficients. These corrections should be calculated with the new value of the proton charge radius [37]. An intriguing question would be to establish how the reported duality of the experimental value of this quantity is reflected in the FSI corrections.

\subsection{Depolarization by multiple Coulomb scattering}

Polarized electrons from neutron decay undergo multiple scattering (mainly at very small angles) in the rest gas of the decay chamber, $p-e$ converter foil, vacuum window, helium interface, separating Mylar foil, He/isobutane gas mixture of MWDC and in the Mott target $(\mathrm{Pb}$ or $\mathrm{U}$ film). In each individual scattering, the original momentum is changed and electron spin can be rotated by internal magnetic field. The traversed materials are amorphous or microcrystalline with randomly oriented internal fields so 
that the net effect of multiple scattering are the energy loss, angular straggling and depolarization. By far the largest depolarization occurs in the Mott target since the scattering cross section scales with the squared atomic number of material. The theory of these effects exists $[38,39]$ so that reliable corrections can be calculated using transport codes. In the nTRV experiment [7,20-22], the systematic uncertainty of this correction was on the level of a few times $10^{-4}$. The largest ingredient to this uncertainty arises from the lack of experimental data in the energy range $0.1-0.8 \mathrm{MeV}$ needed for validation of the Monte-Carlo transport code so that we adopted the worst case difference. We are confident that with a dedicated calibration experiment similar to the one described in Ref. [40] and accurate mapping of the Mott target thickness (cf. [41]) one can bring the systematic uncertainty of the electron depolarization correction to a level of a few times $10^{-5}$.

\section{3. " $g-2$ effect"}

Momentum and spin vectors of an electron traversing external magnetic field undergo precession at slightly different frequencies (cyclotron vs. Larmor frequency) since the $g$-factor is not exactly equal 2 . In consequence, the perpendicular (to the field lines) component of the precessing electron spin vector acquires with respect to momentum approx. $7 \mathrm{mrad}$ of additional phase per revolution. This effect can be corrected for knowing the magnetic field distribution and the path of electron. In BRAND, the external neutron spin guiding field distribution will be mapped and the electron tracks will be directly measured so that the precise calculation of the corrections is straightforward. If the guiding field strength is smaller than $0.5 \mathrm{mT}$, the correction can be neglected. The immunity of the BRAND setup against the " $g-2$ effect" makes it unique and complementary to the ongoing and planned neutron decay experiments utilizing strong magnetic field (in the range of a few $\mathrm{T}$ ) for separation and guiding charged decay products towards detectors. Such experiments do not measure the decay origin so that the desynchronization between electron momentum and spin vectors cannot be followed and corrected for.

\subsection{Momentum rotation in external electric field of $p-e$ converter}

The detection of recoil protons requires their acceleration in the external electric field to about $20-30 \mathrm{keV}$. In our scenario, accelerated protons will eject secondary electrons from a $p-e$ converter foil. Subsequently, electrons will be accelerated towards a plastic scintillator. The $p-e$ conversion scenario for proton detection is chosen by purpose because the electric field barrier will be unavoidably crossed also by beta electrons. If the electric field barrier would be asymmetric, the electrons would experience not only additional energy change but also they would acquire additional phase between momentum and spin vectors. The effect is large: e.g. for electrons with $100 \mathrm{keV}$ kinetic energy entering the potential barrier of $30 \mathrm{kV}$ at $45^{\circ}$ incident angle (resp. field direction), the additional phase at the barrier top will be as large as $12^{\circ}$. For the symmetric barrier, as planned in BRAND, the deflection of the electron momentum is compensated up to the second order effects due to possible local non-uniformities of the electric field. This remnant will be further suppressed by symmetry of the detector arrangement: the numbers of events with negative and positive deflection angle will be about equal. If necessary, adequate corrections can be evaluated with high precision.

\subsection{Momentum rotation in external electric field of MWDC}

The systematic effect of the electric field generated by the sense wires of MWDC is at least an order of magnitude smaller than that of the $p-e$ converter since the applied voltage does not exceed $2 \mathrm{kV}$. Symmetric illumination (left vs. right side of the field wire) suppresses the effect in the same way as described in 4.4. Further suppression is caused by fine granulation of MWDC and additional randomization due to multiple Coulomb scattering effects in the MWDC gas. Therefore the MWDC electric field will effectively account only to the electron depolarization. It is planned to perform an independent calibration experiment using a polarized electron beam as explained in 4.2 at later project stage when depolarization effects will start dominating the systematics.

\section{Simulations}

Transport of protons and electrons (both primary and secondary originating from the $p-e$ converter foil) was investigated with the help of COMSOL [35] simulation package. By applying special configuration of electrodes, recoil protons are focused on a small area of the converter foil. Similarly, the secondary electrons are perfectly collected by "proton" detectors. As a result, the distributions of the deflection angle were obtained. Figure 5 presents a few results from that simulation. The worst case detection efficiency of protons (including $p-e$ conversion losses) is higher than $90 \%$. The detection efficiency of primary electrons from neutron decay is essentially defined by the overall geometrical transparency of the vacuum window and should be close to $50 \%$.

In the first approach, rotationally symmetric structure for 216 module windows arranged in 36 rows at $10^{\circ}$ angular step (cf. Fig. 6a) and featuring 50\% transparency was proposed. In order to check mechanical deformations and stability of such a vacuum chamber made of $10 \mathrm{~mm}$ thick aluminum tube and having the outer diameter of $350 \mathrm{~mm}$ and the total length of $1500 \mathrm{~mm}$ the ANSYS engineering simulation software [36] was used. The calculations show that this design can sustain external overpressure of 1.3 bar with a high safety factor. The maximum stress obtained was $86 \mathrm{MPa}$ which is much smaller than the yield strength value of $276 \mathrm{MPa}$ for aluminum alloy used in this simulation. The same conclusion follows from the calculated load multiplier for buckling which in all cases was larger than 5 . The maximum deformation was only about $0.3 \mathrm{~mm}$ (cf. Fig. 6b).

\section{Research and development}

\subsection{Electron gas tracker}

The successful development of a prototype low mass MWDC that operates with He/isobutane gas mixture at 

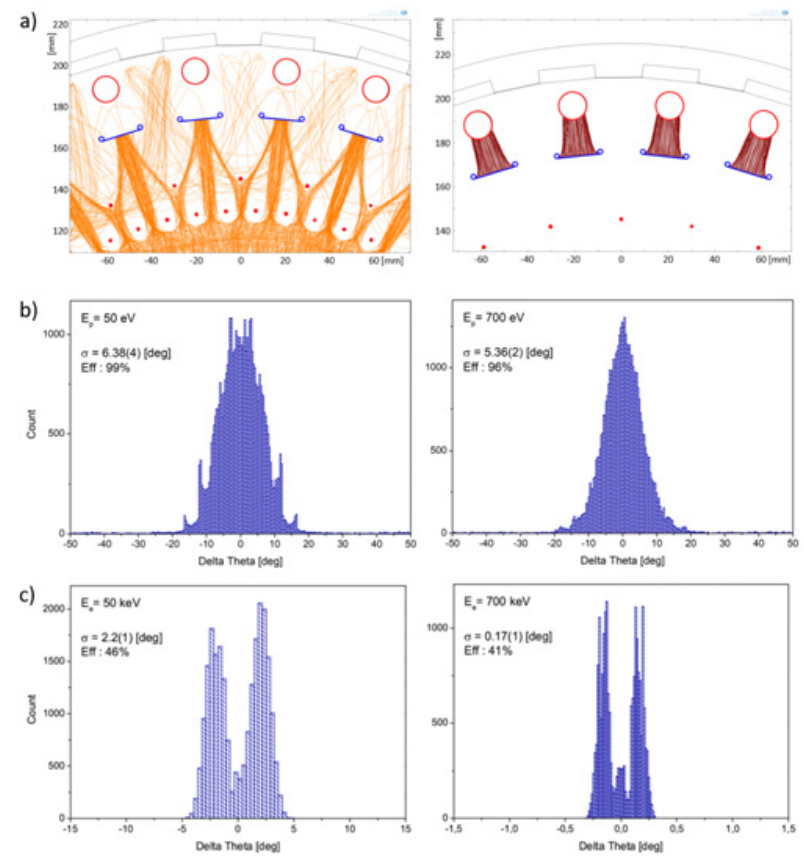

Figure 5. Row a) shows on the left panel the simulated proton tracks hitting the $p-e$ converter foil. $50 \mathrm{eV}$ protons arrive from the field free region and are accelerated in the electric field of $10 \mathrm{kV} / \mathrm{cm}$. Right panel presents the secondary electrons emitted from the $p-e$ converter and accelerated to $30 \mathrm{keV}$ by electric field. Proton detectors are marked as red circles. Rows b) and c) show distributions of the deflection angle (from original direction) for protons and decay electrons, respectively. Only two cases (for boundary energies) are presented.

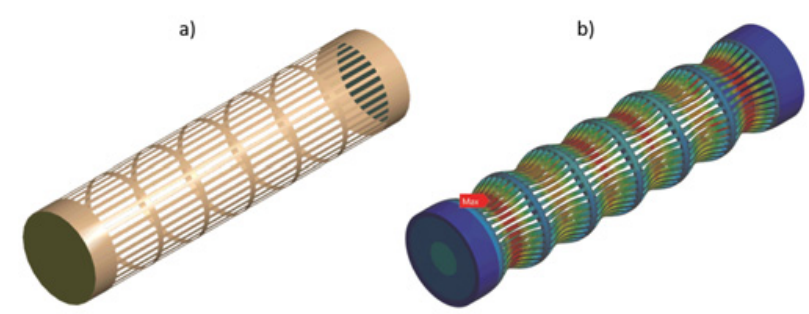

Figure 6. (a) Design of the decay chamber and vacuum window support structure. (b) Simulated with ANSYS software [36] deformation caused by overpressure of 1.3 bar. Red marked area is maximally deformed by $0.32 \mathrm{~mm}$. For better visibility, the transverse deformations were exaggerated in the plot.

reduced pressure down to 300 mbar (miniBETA project [42]) allows for safe planning of a 3D electron tracker that fits optimally to the cylindrical setup geometry. The miniBETA detector consists of very few wires (in average 2 field wires per 1 sense wire at $1 \mathrm{~cm}$ distance) arranged in hexagonal geometry that are readout from both ends. In this way, $x$ - and $y$-components of the track hit position are deduced from the drift time while the $z$-component is obtained using the charge division method. For Mott scattered electrons, the effective optical transparency of miniBETA configuration will be about $92 \%$ as compared to $75 \%$ in the nTRV experiment. For decay electrons, the material composition and total mass passed prior to detection will be comparable to the nTRV setup while the (a)

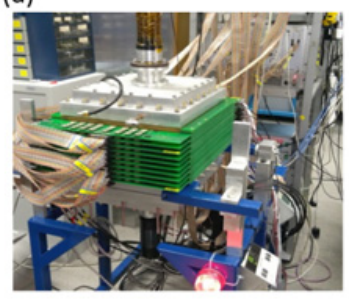

(c) From charge division

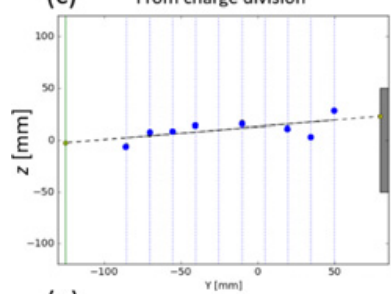

(e)

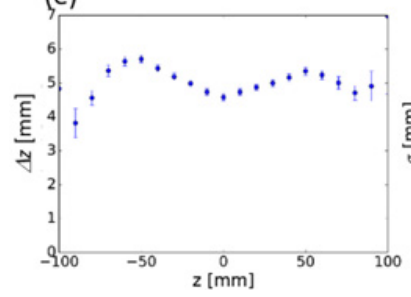

(b)

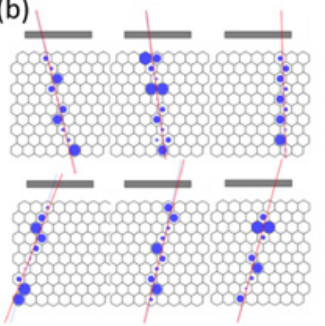

(d) Efficiency $=98 \%, r \in(0,7) \mathrm{mm}$
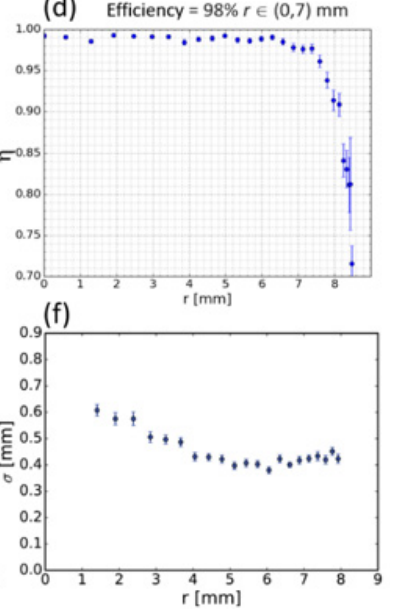

Figure 7. (a) Spectrometer miniBETA - low pressure 10plane MWDC in hexagonal geometry. (b) Typical registered tracks of electrons traversing MWDC projected onto the plane perpendicular to wires. Cells are defined by grounded field wires at the hexagon corners while sense wires are located at hexagon centers. Radii of blue circles represent the shortest distance of the tracks from the sense wire deduced from the drift time. Blue (red) lines show estimated (fitted) tracks. Grey rectangles stay for triggering scintillator. (c) Electron track projected onto the plane parallel to wires deduced from charge division at wire ends. Dashed line is a fit. (d) Single cell efficiency as a function of the distance from the sense wire. (e) Position resolution along wire direction obtained with the charge division method. (f) Position resolution across wires obtained with the drift time.

Mott scattered electrons will experience two times less stopping power. Figure 7a shows a photo of the miniBETA spectrometer. Figures $7 \mathrm{~b}$ and $7 \mathrm{c}$ display typical tracks of electrons traversing 10 wire planes projected onto the planes perpendicular and parallel to wires, respectively. A typical dependence of the cell efficiency as a function of the distance of the electron track from the sense wire is plotted in Fig. 7d showing that the efficiency exceeds $98 \%$ in almost entire cell. Bottom part of Fig. 7 presents the position resolution functions obtained from charge division method (e) and from drift time (f).

\subsection{Vacuum window}

On their way to the detector, electrons from neutron decay will penetrate a window separating the decay chamber vacuum from the gas tracker operating at ambient pressure. On the one hand, it should be as thin as possible in order to minimize energy loss and depolarization, on the other hand, the window must ensure acceptably low permeability for the tracker gas mixture, especially its quencher and, simultaneously, be safe against fracture. The vacuum window and pumping system is being designed to 


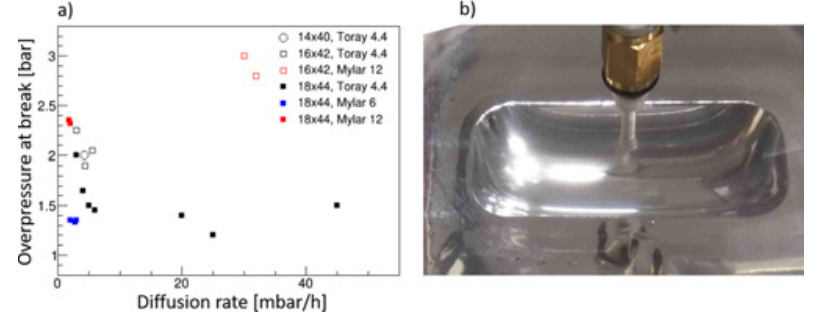

Figure 8. (a) Test results showing the dependence between the beak point pressure and diffusion rate at one bar overpressure for a few different window sizes and foil materials. (b) Window deformation in the test setup.

provide at about $10^{-6}$ mbar pressure in the decay volume (mainly helium penetrating from the tracker). It should be mentioned that some content of helium in the decay chamber would be "healthy" for high voltage, on the other hand, scattering of low energy protons should be avoided. The compromise has to be found. We studied several candidate materials, thicknesses and window opening dimensions. Figure 8a shows the dependence of the diffusion rate as a function of overpressure for different combinations of materials, foil thicknesses and frame dimensions. As a result, a modular design with four, $17 \times 44 \mathrm{~mm}^{2}$ openings placed in a raw and covered with double-side aluminized, $12 \mu \mathrm{m}$ thick Mylar foil has been selected as the current choice. Taking into account a large deformation of the foil (cf. Fig. 8b), its effective thickness is well below $10 \mu \mathrm{m}$. Beside mechanical stability, this selection ensures also very good tightness.

Another attractive material is $4.4 \mu \mathrm{m}$ thick Aramid film from Toray [43], however, it is not commercially available in a double-side aluminized form. Further tests are ongoing.

\section{3. "Proton" detector}

The key features of the "proton" detector should exhibit the following features: (i) Particle identification capability the detector must distinguish between recoiling protons and decay electrons - which means here: between electron bunches consisting of several low energy (approx. $25 \mathrm{keV}$ ) electrons and single higher energy $(>150 \mathrm{keV})$ electrons. (ii) Good timing - it must be fast enough as it will participate in the time-of-flight measurement with the resolution of a few ns. (iii) "Proton" detector must be position sensitive with the resolution of about $10 \mathrm{~mm}$ to allow for reconstruction of proton energy in the decay origin. (iv) "Proton" detector should be robust as it will operate in high electric field.

As the first choice we proposed a thin $(25 \mu \mathrm{m})$ plastic scintillator layer deposited on a lightguide rod made of PMMA. The scintillation light will be readout by a chain of SiPM sensors distributed along the lightguide. The hit position will be deduced from the light distribution on the neighbouring sensors close to the light origin as explained in Fig. 9a. The concept was tested in the laboratory with promising results as shown in Figs. $9 \mathrm{~b}$ and $9 \mathrm{c}$. Figure $9 \mathrm{~b}$ presents the correlation between the evaluated and true hit position while Fig. 9c shows cross sections of the former distribution along a few selected source positions. In this test, the light flashes of the electron bunches were simulated by adjusting the energy deposition of single
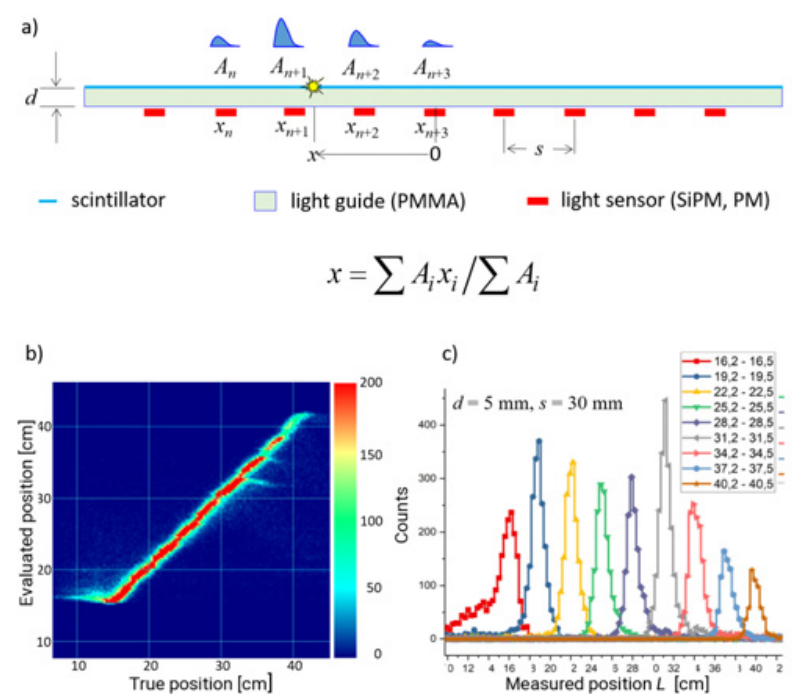

Figure 9. (a) Concept of "proton" detector. (b) Correlation between the evaluated and true hit position measured with a prototype setup as explained in the text. (c) Cross sections of the former distribution along a few selected source positions.

electrons from the radioactive source ${ }^{90} \mathrm{Sr}$. To this end, $1 \mathrm{~mm}$ thick plastic scintillator was used. For the lightguide thickness of $5 \mathrm{~mm}$ and $30 \mathrm{~mm}$ distance between adjacent light sensors, we obtained the average position resolution $\sigma \approx 6 \mathrm{~mm}$. Further optimization of geometry, electronics and used evaluation algorithms is ongoing.

\section{Summary}

BRAND is a complicated and challenging experimental proposal in the field of particle physics at the precision frontier. If successful, it will provide exclusive empirical data for searches of feeble traces of forces potentially contributing to week interactions at the energy scale of several TeV. It will complement other approaches like precision beta spectrum measurements in the neutron and nuclear decays and direct searches performed and planned at high energy colliders. The added value of the expected BRAND results is generated by similar statistical sensitivity and completely different systematics as compared to the "classical" neutron and nuclear beta decay experiments.

In order to mitigate risks, we are assembling a modular and handy setup allowing for testing and optimizing the system components. The most elaborated element of the apparatus exists already and is fully functional (miniBETA spectrometer). The test experiment will be installed on the PF1b beamline at ILL, Grenoble. The primary goal for measurements using the test setup is the systematic characterization and optimization of the neutron beam and its interaction with the BRAND components. The results from this research will actually fix two still open parameters of the regular BRAND polarimeter. The achieved level of the beam halo will define the optimal diameter of the decay chamber while the beam divergence will limit the polarimeter length.

Upon successful completion of the test phase (called "BRAND 0") the full setup will be constructed and installed on the PF1b beamline at ILL, Grenoble and will take data at ambient pressure in the electron tracker. 
In further perspective, the experimental setup will be enclosed in a tank allowing for the tracker operation at reduced pressure. Furthermore, it is planned to instal the BRAND apparatus at ESS, Lund and take advantage of the high intensity pulsed cold neutron beam at the ANNI beamline [44].

This work has been supported in part by The National Science Centre, Poland, under the grant No. UMO-2018/29/B/ST2/02505. Calculations using the COMSOL Multiphysics ${ }^{\circledR} 4.3$ software were performed at the Academic Computer Centre CYFRONET AGH, Cracow, on the PlatonU3 platform.

\section{References}

[1] V. Cirigliano et al., Nucl. Phys. B 830, 95 (2010)

[2] T. Bhattacharya et al., Phys. Rev. D 85, 054512 (2012)

[3] V. Cirigliano et al., JHEP 1302, 046 (2013)

[4] M. Gonzalez-Alonso et al., Ann. Phys. 525, 600 (2013)

[5] M. Gonzalez-Alonso et al., Phys. Rev. Lett. 112, 042501 (2014)

[6] T. Bhattacharya et al., Phys. Rev. Lett. 115, 212002 (2015)

[7] K. Bodek et al., Physics Procedia 17, 30 (2011)

[8] H. Abele, Prog. Part. Nucl. Phys. 60, 1 (2008)

[9] S. Baessler et al., J. Phys. G Nucl. Part. Phys. 41, 114003 (2014)

[10] A.R. Young et al., J. Phys. G: Nucl. Part. Phys. 41, 114007 (2014)

[11] A.N. Ivanov et al., Phys. Rev. C 95, 055502 (2017) and references therein

[12] A.N. Ivanov et al., Phys. Rev. C 98, 035503 (2018) and references therein

[13] J. Jackson et al., Phys. Rev. 106, 517 (1957)

[14] J. Jackson et al., Nucl. Phys. 4, 206 (1957)

[15] M.E. Ebel et al., Nucl. Phys. 4, 213, (1957)

[16] C. Patrignani et al. (Particle Data Group), Chin. Phys. C 40, 100001 (2016) and 2017 update

[17] K. Bodek, Acta. Phys. Pol. B, 47, 349 (2016)

[18] S. Chatrchyan, et al. [CMS Collaboration], JHEP 1208, 023 (2012)

[19] V. Khachatryan, et al. [CMS Collaboration], CERN Rep. CMS-PAS-EXO-12-060, (2013)
[20] A. Kozela et al., Phys. Rev. Lett. 102, 172301 (2009)

[21] G. Ban et al., Nucl. Instrum. Methods Phys. Res., Sect. A 565, 711 (2006)

[22] A. Kozela et al., Phys. Rev. C 85, 045501 (2012)

[23] W.S. Wilburn et al., J. Res. NIST 110, 389 (2005)

[24] B. Maerkisch et al., Nucl. Instr. Meth. Phys. Res. A 611, 216 (2009)

[25] D. Dubbers et al., Nucl. Instr. Meth. Phys. Res. A 596, 238 (2008)

[26] O. Zimmer et al., Nucl. Instr. Meth. Phys. Res. A 440, 548 (2000)

[27] F.E. Wietfeldt et al., Nucl. Instr. Meth. Phys. Res. A 611, 207 (2009)

[28] B. Tipton et al., AIP Conf. Proc. 539, 286 (2000)

[29] R. Alarcon et al., URL http://nab.phys. virginia.edu/ nab_loi.pdf, Letter of intent (2005)

[30] H.P. Mumm et al., Rev. Sci. Instr. 75, 5343 (2004)

[31] T. Soldner et al., Phys. Lett. B, 581, 49 (2004)

[32] S. Hoedl et al., J. Appl. Phys. 99, 084904 (2006)

[33] J. Sromicki, Nucl. Instr. Meth. Phys. Res. A 440, 609 (2000)

[34] T.M. Ito et al., Nucl. Instr. Meth. in Phys. Res. A 571, 676 (2007).

[35] COMSOL Multiphysics ${ }^{\circledR}$, https://www.comsol. $\mathrm{com} /$

[36] ANSYS engineering simulation software, https : //www. ansys. com/

[37] C. Patrignani et al. (Particle Data Group), Chin. Phys. C 40, 100001 (2016) and 2017 update

[38] N. Sherman, Phys. Rev. 103, 1601 (1956)

[39] F. Salvat et al., Comput. Phys. Comm. 165, 157 (2005)

[40] J. Sromicki et al., Phys. Rev. Lett. 82, 57 (1999)

[41] A. Kozela et al., Nucl. Inst. and Meth. in Phys. Res. B 102, 172301 (2010)

[42] M. Perkowski et al., Acta Phys. Polonica B 49, 261 (2018)

[43] Toray Industries Inc.

[44] E. Klinkby, and T. Soldner, J. Phys: Conf. Ser. 746, 012051 (2016), T. Soldner et al., EPJ Web of Conf. 219, 10003 (2019) 